07.X-OI MATERIAIS NTTH VALENCE INSTABILITIES. E. Kaldis, Laboratorium für Festkörperphysik, ETHZ, 8093 Zürich, Switzerland

Materials with valence instabilities are small gap semiconductors (e.g. SmS, $\mathrm{Sm}_{3} \mathrm{~S}_{4}$ ) or metals (e.g. TmSe, CeN) which under pressure may change the valence state of their cation. Although this phase transition is followed by a large volume contraction (approx. 10 \%), no change of the structure (NaCl) takes place. The change of valence can be achieved in TrSe also by variation of nonstoichiometry ( $E$. Raldis et al $J$. Phys. 4o (1979) C5-366) or mixed crystal formation

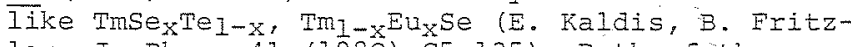
ler, J. Phys. 41 (1980) C5-135). Both of these. parameters lead to changes of the crystal field splitting of the 5d-band and therefore vary the degree of overlapping of the $5 d-b a n d$ with the 4f localized level.

Solution calorimetry (TmSe, $\mathrm{Sm}_{3} \mathrm{~S}_{4}$ ) and $\mathrm{T}-x$ phase diagram studies (TmSe, $\mathrm{TmSe}_{1-\mathrm{xTe}}, \mathrm{Sm}_{3} \mathrm{~S}_{4}$ ) indicate that valence instabilities introduce phase instabilities. The thermodynamically unstable phases show, however, appreciable metastability. Calorimetric measurements in CeN show that alloying with less than 1 o at. oxygen increases the stability (decrease of the heat of solution) by approx. 30 \%. This effect is probably due to a destruction of the unstable mixed valence state of CeN by the oxygen doping.

\section{X-02 FINE METAL CRYSTALLITES: \\ MORPHOLOGY AND STRUCTURE}

By Ryozi Uyeda.

Department of Physics, Meijo University Tempaku-ku, Nagoya, Japan

Fine metal crystallites of diameter less than I $\mu \mathrm{m}$ can be produced by the evaporation and subsequent condensation of a metal in an atmosphere of inactive gas. A study of these crystallites by electron microscopy and electron diffraction was initiated in 1962 by the present author and pursued in Nagoya University and Meijo University. Up to the present most of the ordinary metals and semi metals (30 elements) have been studied $[1,2]$.

Some of the important results are as follows: Metal crystaliites produced by the condensation in the gas look like a smoke. Those of crystallographic interest are produced at gas pressures between 10 - 100 Torr. A typical smoke has a shape like a candle flame, consisting of inner, intermediate and outer zones.

The mechanism of crystal growth in a smokew. studied. A remarkable result is that the crystallites grow not only by condensation of vapour, but also by collision and subsequent coalescence of crystallites.
Since crystallites grow in a free atmosphere without any substrate, those grown under appropriate conditions, which are single crystals and twins, show beautiful crystal habits: rods, plates or polyhedra.

Among them truncated octahedra, rhombic dodecahedra and hexagonal prisms are approximately the equilibrium forms respectively of fcc, bcc and hcp. They are produced in the intermediate zone of a smoke.

The so-called multiply twinned particles [3], icosahedra and pentagonal decahedra, are found for fcc metals and Ge. They have no crystallographic symmetry and are better to be called large metal molecules.

Metals with phase transformation show habits belonging to more than two phases. The external shape is preserved when the inner structure transforms from a high temperature phase to a low temperature phase.

New structures special to fine particles are found for $\mathrm{Cr}, \mathrm{Mn}$ and $\mathrm{Ge}$. That for $\mathrm{Cr}$ is identified as A-15 type [4], while the others are not yet analysed.

A number of slides will be projected in the talk to show beautiful crystal habits.

[1] R. Uyeda, J. Cryst. Growth, 24/25 (1974) 69

[2] R. Uyeda, J. Cryst. Growth, $\frac{45}{45}$ (1978) 485

[3] Sh. Ogawa and Sh. Ino, Advances on Epitaxy and Endotaxy (VEB Deutscher Verlag, Leipzig, 1977) 705 [4] K. Kimoto and I. Nishida, Thin Solid Films, 17 (1973) 49

07.X-03 NECHANISMS OF EPITAXY

By R. Kern, C.R.M.C. - CNRS, Campus de Luminy, case $913-13288$ MARSEILLE CEDEX 9 , FRANCE.

The understanding of epitaxy has received a great impulse by extensive use of surface physics methods. The methods are briefly considered and their practical power analysed. In-situ methods have received special attention in recent years and very original results have been obtained.

Ellipsometry is such a method which is able to give simultaneously information about thickness and composition inside an industrial epitaxy reactor. Some typical examples will be given on systems as metal - insulator, metal - semiconductor, semiconductor - semiconductor. 